\title{
BUSINESS MODELS FOR CLOUD COMPUTING: EXPERIENCES FROM DEVELOPING MODELING \& SIMULATION AS A SERVICE APPLICATIONS IN INDUSTRY
}

\author{
Tamas Kiss \\ Huseyin Dagdeviren \\ Centre for Parallel Computing \\ University of Westminster \\ 115 New Cavendish Street \\ London, W1W 6UW, UNITED KINGDOM
}

\author{
Simon J. E. Taylor \\ Anastasia Anagnostou \\ Department of Computer Science \\ Brunel University London \\ Kingston Lane \\ Uxbridge, UB8 3PH, UNITED KINGDOM
}

\author{
Nicola Fantini \\ CloudBroker $\mathrm{GmbH}$ \\ Räffelstrasse 25 CH-8045 \\ Zurich, SWITZERLAND
}

\begin{abstract}
The potential of cloud computing is gaining significant interest in Modeling \& Simulation (M\&S). The underlying concept of using computing power as a utility is very attractive to users that can access stateof-the-art hardware and software without capital investment. Moreover, the cloud computing characteristics of rapid elasticity and the ability to scale up or down according to workload make it very attractive to numerous applications including M\&S. Research and development work typically focuses on the implementation of cloud-based systems supporting M\&S as a Service (MSaaS). Such systems are typically composed of a supply chain of technology services. How is the payment collected from the enduser and distributed to the stakeholders in the supply chain? We discuss the business aspects of developing a cloud platform for various $M \& S$ applications. Business models from the perspectives of the stakeholders involved in providing and using MSaaS and cloud computing are investigated and presented.
\end{abstract}

\section{INTRODUCTION}

Cloud computing is a paradigm for distributed computing that, according to the widely accepted definition provided by the US National Institute of Standards and Technology (NIST), provides a "ubiquitous, convenient, on-demand network access to a shared pool of configurable computing resources (e.g., networks, servers, storage, applications, and services) that can be rapidly provisioned and released with minimal management effort or service provider interaction" (Mell and Grance 2011; NIST 2013). In Modeling \& Simulation (M\&S) cloud computing has the potential to give flexible service provision as well as scalable and reliable computing power without initial capital investment to facilitate computation intensive simulations. The concept of Modeling \& Simulation as Service (MSaaS) therefore enables simulation software vendors and providers to offer simulation applications through the cloud to their endusers. The user can consume simulation services without having to install or maintain the simulation software locally. The solution can also scale up seamlessly as the number of end-users and/or the complexity of the simulation tasks increase. 


\section{Kiss, Dagdeviren, Taylor, Anagnostou, and Fantini}

Most of the related research and development effort in this area focuses on the development of cloudbased infrastructures to provide MSaaS. However, once a service is deployed and available, how should a user pay for that service and how should that revenue be distributed across the various stakeholders that support the provision of that service? What business models should be used? The European FP7 Cloudbased Simulation Platform for Manufacturing and Engineering (CloudSME) project (www.cloudsme.eu) aims to simplify the deployment of MSaaS on different clouds and has developed MSaaS solutions across several M\&S domains. In this paper, we share our practical experiences in creating several business models that account for the different stakeholder perspectives that exist in this "supply chain" of technology from the end-user to the cloud infrastructure provider. Our paper is structured as follows. We first give a brief orientation on Cloud Computing and define MSaaS. To give a context to our experiences, we then present our CloudSME project and cloud-based simulation platform. A business model for MSaaS is then presented and then developed into more detailed business models reflecting the needs of different stakeholders involved in the consumption and provision of services. The paper then concludes with a short discussion on the need for a simple mechanism for charging and revenue distribution via one-stop-shops or similar mechanisms to support business models for cloud computing.

\section{CLOUD COMPUTING}

As presented in Taylor, et al. (2014b), building on the above NIST definition, the main concept of cloud computing is elasticity. Users and applications can instantly access and release on-demand computational assets, storage, other service applications, etc. depending on their changing needs. These resources are provided on the basis of different types of clouds that reflect the organizational structure of the cloud provider(s) and user(s). These include Private clouds (accessed only by the users of a single organization via private networks), Community clouds (an extension of a private cloud accessed by more than one organization with common interests where resources are located on or off site and can belong to one or more organizations within the community or a third party (e.g., a government cloud), Public clouds (accessible by the general public and supported by an infrastructure provider (e.g., the Amazon Elastic Compute Cloud (EC2)), and Hybrid clouds (a combination of two or all three of the above).

Cloud use and provision can be organized on the basis of cloud computing service models. There are three defined main service models. These are Infrastructure as a Service (IaaS), Platform as a Service (PaaS), and Software as a Service (SaaS). Cloud consumers (users) typically can access all three services via a web interface or some language supported API. IaaS allows access to a virtual infrastructure mapped to physical hardware where the user can deploy operating systems and application software. PaaS stands on top of the IaaS and facilitates software deployment, configuration and management. PaaS users can deploy existing software or develop software with libraries and compilers provisioned by the cloud provider. The most commonly used service model is SaaS, which sits atop the PaaS. A SaaS user has only application level access. Users can use the provided applications deployed as online services with no knowledge of the platform or the infrastructure, accessing elastically provided resources on demand.

\section{MODELING \& SIMULATION AS A SERVICE (MSaAS)}

Could computing has two principle features that could be attractive for M\&S. The first is service provision. Deploying a simulation application as a service on a cloud means that users can easily access that service when required. Conceptually, this is similar to deploying an application on a web server. However, the difference is that cost is only incurred when the service is used, rather than an "always on" web server. Further, if the service is deployed appropriately, then the service could be provided on an elastic basis. The service provider should be able to easily upscale the processing power of their application or create multiple versions of their service on demand. The second attractive proposition is the access to on demand elastic high performance computing. Many M\&S applications run simulations of the same model with different parameters. These experiments can be highly time consuming. As these runs are independent, simulations can be executed faster by using more computers. This means that time for 


\section{Kiss, Dagdeviren, Taylor, Anagnostou, and Fantini}

experimentation can be reduced significantly (or more experimentation can be done in the same time) by "hiring" more cloud-based computing resources.

Modeling \& Simulation as a Service (MSaaS) is emerging as term that describes the development of cloud-based software services for $M \& S$ applications. In literature, the main focus has been on the technological aspects of cloud development and provision. Cayirci (2013) reviews technological issues relating to the direct deployment of MSaaS on a cloud including security, risk, and service composition. Other authors address technological architectures that have elements of cloud-based services supporting M\&S. For example, Rossetti and Chen (2012) present a prototype architecture that directly deploys simulation on a cloud to support supply chain networks with limited parallel computing. Wu, Qiao and Poon (2014) propose an architecture for cloud manufacturing in the semi-conductor industry that combines collaboration services underpinned by business intelligence, cloud, resource and data layers. This focuses on interoperating services to support manufacturing (operations, logistics, procurement, etc.) and links to potential MSaaS. This is similar in concept to cloud-based architectures being developed by the CloudFlow project (www.eu-cloudflow.eu). Tolk and Mittal (2014) raise the issue of a lack of work on the conceptual foundations of MSaaS as highlighted in the series of Grand Challenges in M\&S panels (Taylor et al. 2012, 2013a, 2013b) and advocate the novel concept of Mobile Propertied Agents that could bring together key issues needed for MSaaS. Johnson and Tolk (2013) raise the work by NATO on cloudbased simulation with respect to technical, governance, security, business model and conceptual perspectives. NATO work continues with the activities of the NATO Modeling and Simulation Group MSG-131 ("Modelling and Simulation as a Service: New Concepts and Service Oriented Architectures") (Siegfried, et al. 2014). This group are investigating MSaaS from national (defense) perspectives and experiences as part of a developing "NATO M\&S as a Service Concept" under NATOs Allied Command Transformation (ACT). In the sense of M\&S as being a key enabler for military training, analysis and decision making, they define MSaaS as "M\&S as a Service (MSaaS) is a means of delivering value to customers to enable or support modelling and simulation (M\&S) user applications and capabilities as well as to provide associated data on demand without the ownership of specific costs and risks." "Value" is determined by what the service enables the customer to do (e.g., a professional service such as Verification \& Validation) or an IT or technical service (e.g., a weapon effects service integrated within a simulation environment). They conclude that MSaaS is an architectural and organizational approach that promotes abstraction, loose coupling, reusability, composability and discovery of M\&S services with the objective of effectively and efficiently supporting operational requirements (e.g., executing an exercise) and to improve development, operation and maintenance of $M \& S$ applications.

We consider the NATO definition to be general purpose enough to "fit" the use of M\&S across many domains, not just the military. A user application could be a COTS Simulation Package such as the discrete-event simulation software SIMUL8 or the ASCOMP's computational fluid dynamics software TransAT. A capability could be how the simulation software is used to solve a problem (such as building and experimenting with a model of a manufacturing system) or to provide a service such as 3D modeling online. Associated data could be simulation input data or simulation output results. The definition does reflect the cost and risk of ownership and support but not the advantages of cloud elasticity. However, as this is a service definition then this aspect of cloud computing could be considered as an MSaaS capability (i.e., not all MSaaS applications need elasticity). Value follows a similar line of reasoning as it is left as the provision of a service. The conclusions are similarly general except that requirements would reflect the domain of application. However, linking these concepts of MSaaS value to cloud computing and then to the mechanisms of charging and revenue distribution requires further investigation and expansion. In the next section we provide a brief overview of our project and industrial case studies to give the context and background to our business models. 


\section{Kiss, Dagdeviren, Taylor, Anagnostou, and Fantini}

\section{THE CLOUDSME PROJECT AND PLATFORM}

The main aim of the CloudSME project is to help simulation companies and end-users to develop complex cloud computing solutions for MSaaS by providing supporting technologies to simplify this process. The main technology is the CloudSME Simulation Platform (CSSP) (shown in Figure 1), a PaaS that supports service provision, High Performance Computing (HPC) and multi-cloud deployment (Taylor, et al. 2014a). The project is industry-based and focuses on four key areas of M\&S: Computational Fluid Dynamics, process (discrete-event) simulation, 3D modeling and data analytics. The University of Westminster (UK) is the project coordinator with Brunel University London (UK) coordinating the industrial use cases. The domains covered by the project include oil and gas industries, engine design and manufacturing, UAV design, general purpose manufacturing, food supply chain, port logistics, shoe insole manufacturing, and inventory control. The CSSP uses multiple IaaS clouds provided by MTA SZTAKI (Hungary), BIFI (Spain), University of Westminster (UK), CloudSigma (Switzerland) and Amazon.

In the Cloud Platform Layer, the CSSP architecture combines the WS-PGRADE/gUSE (Grid User Support Environment) developed by MTA SZTAKI (guse.hu) (Kacsuk, et al. 2012) and the multi-cloud CloudBroker Platform (CBP) developed by CloudBroker, $\mathrm{CH}$ (www.cloudbroker.com) to create a platform for cloud-based HPC (a PaaS). Software requiring cloud deployment and/or HPC is redeveloped on the CSSP as SaaS, hosted by the CSSP PaaS and uses clouds made available through IaaS. Developers can therefore create rapidly their scalable SaaS applications using the CSSP across one or more different clouds and can use cloud-based HPC where appropriate. The CSSP offers several options to cloud-based application development.

WS-PGRADE/gUSE supports the development and deployment of HPC applications across different types of Distributed Computing Infrastructures (DCIs) such as clusters, institutional desktop grids and clouds. It is widely used to create HPC applications for a wide range of scientific communities (see www.sci-bus.eu and Kacsuk (2014)). WS-PGRADE/gUSE consists of three tiers: a Presentation tier, a Middle tier and an Architectural tier. The Presentation tier consists of WS-PGRADE (Web Services Parallel Grid Runtime and Developer Environment Portal). It has a graphical workflow editor that allows users to create and populate HPC workflows for their applications that run on various DCIs (including clouds). Once a workflow is created, it is saved and managed in the Middle tier gUSE services. In the Architectural tier, gUSE uses the DCI-BRIDGE job submission service to submit cloud jobs to the CBP and other DCIs.

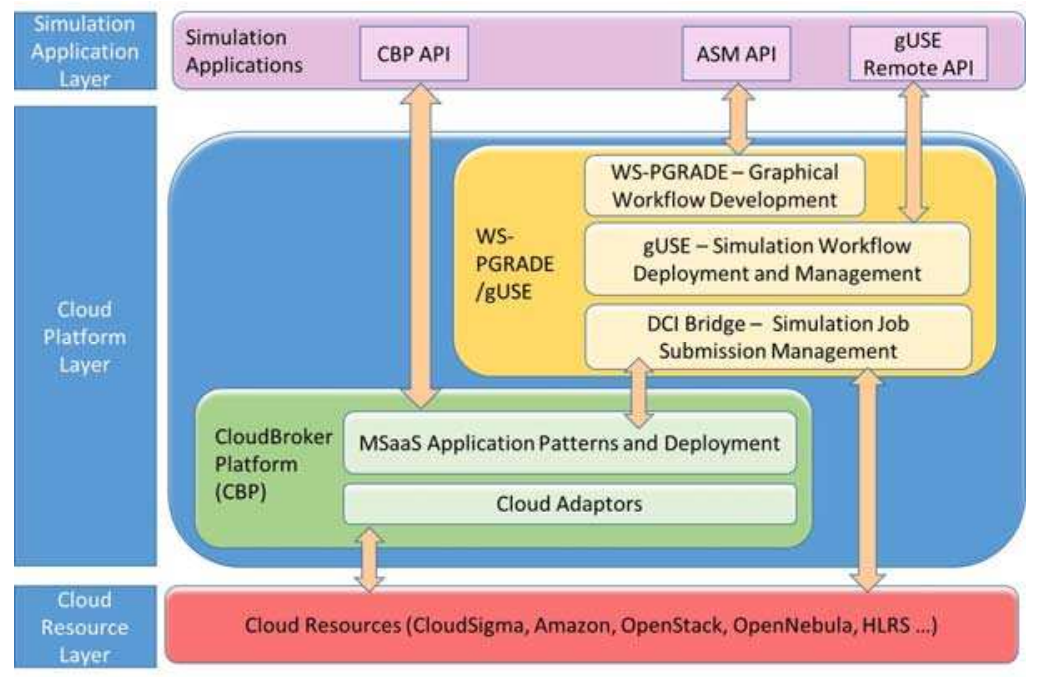

Figure 1: The CloudSME Simulation Platform Architecture. 


\section{Kiss, Dagdeviren, Taylor, Anagnostou, and Fantini}

A "typical" cloud implementation involves software being developed for one cloud (e.g., Amazon). Different clouds offer different functionality and costs. The concept behind the CBP is that it presents a cloud platform that developers can use to manage their implementations across multiple clouds. This means that once an application has been deployed on different clouds, the CBP can help developers deliver a flexible service that enables cloud-based applications to be easily managed (and charged) across product offerings based on different cloud infrastructures. The CBP environment is a web-based application store for the deployment and execution of compute-intensive applications on a cloud and widely automates user, software, resource, job, and invoice management. It is suitable for any kind of batch-oriented command line software, both Linux- and Windows-based, and both serial or parallel processing (via MPI for example). It can be accessed through any web browser and through different application programming interfaces (APIs). Taylor, et al. (2014a) discuss how the CSSP has been used to support agent-based modeling and simulation applications.

\section{CLOUD BUSINESS MODELS AND STAKEHOLDERS}

The CSSP is an example of a PaaS that facilitates access to one or more IaaS. In other words, a simulation company can develop their $M \& S$ software service and run it on a platform service. The platform service coordinates access to the computational resources of the cloud infrastructure provider. Using the different APIs of the Simulation Application Layer, the simulation software developers of the CSSP have developed different approaches to MSaaS deployment that range from single point of use, to scalable services and scalable HPC solutions. These are being presented as "one-stop-shops" that the developers are using as gateways for their users to access their software. Additionally, a generic one-stop-shop called the AppCenter has been created within the CloudSME project to support companies who would like a one-stop-shop type of gateway to their MSaaS solution but do not want to develop one themselves. A new user wanting to use an MSaaS "App" registers and logs into the AppCenter and accesses the App.

Using one of our industrial case studies as an example basis for a business model, EUROBIOS is a French-based company specializing in industrial complexity analysis in a variety of application areas (www.eurobios.com). They are using ASCOMP's TransAT Computational Fluid Dynamic software deployed on different clouds via the CSSP. TransAT is deployed in our AppCenter (but could be part of a dedicated one-stop-shop). The AppCenter means that EUROBIOS' usage of TransAT can be tracked. The cloud platform and infrastructure use is tracked through the CSSP. The commercial relationship is therefore EUROBIOS using ASCOMP's software that uses the CSSP developed by multiple stakeholders that uses (say) Amazon cloud resources. Given that cloud-based solutions can be collections of software, this seemingly complex relationship may well be typical of cloud-based commercial offerings. A business model, incorporating and linking the value chains of all stakeholders is therefore required to deliver MSaaS. Developing this business model is complex and challenging due to a number of factors:

1. The business venture is based on close partnership and collaboration amongst a number of key stakeholders. The multi-stakeholder nature of the CSSP offering adds complexity to the development of the business model as the platform needs to deliver business benefits to all participating stakeholders.

2. The simulation service providers have different offerings ranging from providing simulation software as a service and/or using a simulation software service as part of a consultancy.

3. The stakeholders have varying business requirements for pricing, product delivery, payment methods, etc.

4. End-user consumers have different levels of technical knowledge and therefore need access to MSaaS in different ways.

5. The manufacturing and engineering market (the focus of the CloudSME project) is a rather heterogeneous market in terms of their activities and products. For example, in manufacturing sector the products range include fabricated metal, electrical parts, minerals, food, paper, textile, 


\section{Kiss, Dagdeviren, Taylor, Anagnostou, and Fantini}

furniture and others. The business model should accommodate various scenarios in this heterogeneity and not just on technology delivery (i.e., it should reflect business practices in those domains - some sectors cannot support pay-per-use models for example).

Within the CloudSME project there are four stakeholder groups. Note that most of the companies in the project are SMEs. The stakeholder groups are described below.

End-users: At one end of this chain, there exist the end-user companies from the manufacturing and engineering sector. In the CloudSME project, these companies are represented as two distinct categories: end-users who already utilize simulation technologies and end-users for whom simulation solutions are novel.

Simulation software providers: There are two kinds of simulation software providers. Companies that develop their simulation software as MSaaS and simulation consultancies that use these software services (i.e., simulation consultancies act as multipliers that offer the simulation solutions of various vendors).

Cloud platform providers: Next group in this chain are the cloud platform providers. The cloud platform providers support MSaaS and enable simulation software to access cloud resources seamlessly in a user-transparent way and a PaS solution, which allows simulation software providers or consultant companies to build MSaaS solutions for the end-users. In CloudSME project, the cloud platform providers are namely CloudBroker and SZTAKI which offer the integrated CloudBroker/WS-PGRADE CSSP that serves as the basis for the PaaS solution.

Cloud resource providers: Finally, the simulation platform requires cloud-based hardware resources therefore IaaS cloud resource providers are another group of stakeholders that are essential for the operation of the cloud platform layers. Cloud resource providers will directly benefit from the successful operation of the CloudSME simulation platform as it would increase the usage of their resources.

In the CloudSME project, the CSSP PaaS provider will have two types of customers: a simulation software provider (i.e., simulation software vendor or a simulation consultancy) and manufacturing and engineering simulation end-users. This means manufacturing and engineering simulation end-users will either interact with the CloudSME platform directly or through the simulation software vendor or a simulation consultancy. This interaction model provided the basis for developing the business model as, it not only shows required interactions, but also a clear division of roles between stakeholders.

The next section will present the specific business models that have been developed.

\section{MSaAS BUSINESS MODELS}

In the CloudSME project MSaaS business models were developed iteratively using the input received from all stakeholders (24 companies). This necessitated a number of meetings with the project partners, in groups or individually. Initially a group project meeting took place where initial ideas, requirements and constraints for the business model were brainstormed. This provided the basis for developing the initial business models which were then elaborated and refined in further interactions (i.e., meetings or conference calls) with stakeholders individually or in small groups.

During these interactions, it became apparent that one business model would not suffice due to the flexibility required in terms of cloud use. For example, in addition to offering simulation services to customers on individual basis, a simulation provider wanted to offer 'generic' simulations to a specific industry through templates specifically developed for that specific industry. This led to considering developing multiple business models, each one based on the use of the CSSP and cloud computing for MSaaS. During the discussions, five uses were identified each of which then served as the basis of a business model. These business models are as follows:

1. Specialized "one-stop-shop" for a specific industry

2. Specialized "one-stop-shop" for a specific company 


\section{Kiss, Dagdeviren, Taylor, Anagnostou, and Fantini}

3. Cloud extension to a desktop simulation package or a web-based simulation solution

4. Direct execution of the simulation software from the CloudSME AppCenter

5. The business model of the PaaS provider

These business models were based on the following principles:

1. Offer simulation services as one-stop-shops to specific companies or targeted manufacturing and engineering market segments in order to ensure simplicity for novice simulation users;

2. Enable simulation software vendors to extend their products with cloud access seamlessly to offer existing simulation users to accelerate simulation execution through CloudSME platform if needed for complex simulations;

3. Offer an overall directory for simulation software (shopping mall like) to all manufacturing and engineering SMEs.

\subsection{Specialized One-Stop-Shop for a Specific Industry}

In this model the CloudSME simulation platform customer, typically a simulation software vendor or a simulation consultancy, offers a specialized one-stop-shop MSaaS solution for simulation end-users. A specialized one-stop-shop for a specific industry has the following characteristics:

- Specialized website offered by simulation software vendor or simulation consultancy;

- Readymade simulation applications to be purchased by simulation end-users based on the SaaS model;

- Target audience is from a well-defined industry where a large number of end-users can benefit applications are created using a template approach;

- The one-stop-shop provider charges the end-users and provides full customer support - the onestop-shop provider carries all responsibility when interacting with the customer;

- The simulation runs through the CSSP; and

- Can be advertised via domain specific channels, but can also be directly linked to the CloudSME marketplace.

Figure 2(a) illustrates the "specialized one stop shop for a specific industry" model.

\subsection{Specialized One-Stop-Shop for a Specific Company}

This model can be regarded as a special restricted and closed version of the first model, "specialized onestop-shop for a specific industry". In the current model, a specialized simulation application is developed and operated for one targeted company only as simulation end-user. The rationale behind listing this as a separate model is that some simulation MSaaS offerings could be rather specific and could not be easily reused by multiple companies. In this case, a specialized solution can be developed for the end-user that can only be accessed by the targeted company. The commercial viability of this model may need to be further investigated. However, in case of a "heavy end-user", the model could be financially viable. The operator of the one-stop-shop for a specific company provides direct customer support to its end-users and serves as the only entry point and communication towards the final end-user. The end-user makes a commercial contract only with the one-stop-shop provider. The one-stop-shop provider carries all responsibility when interacting with the customer. As in previous cases, running the simulation through the CSSP is compulsory and an essential requirement. If the simulation does not run through the platform, then it is outside the scope of this business model. Listing the solution in the CloudSME AppCenter is optional. Figure 2(b) shows this business model. 


\section{Kiss, Dagdeviren, Taylor, Anagnostou, and Fantini}

\subsection{Cloud Extension to a Desktop or Web-based Services Simulation Package}

In this scenario, the CSSP customer is a simulation software vendor who extends its desktop or web services simulation product with built-in cloud access via the CSSP to offer MSaaS. The simulation software is packaged (if appropriate), licensed and sold to the simulation end-user in the traditional way. However, the installed product includes direct access to the CSSP and offers the capability to accelerate simulation on the cloud on-demand.

The simulation software vendor provides direct customer support to simulation end-users that covers the traditional functionalities of the product. However, when using the cloud extension, two major scenarios are envisaged: (a) a simulation end-user creates account on the CSSP that manages cloud-based execution. In this case the simulation end-user enters a commercial contract with the CloudSME simulation platform provider. The CSSP provider is responsible for providing direct customer support for the end-user; (b) the simulation software vendor is the only entity who has an account on the CSSP, and all cloud-based execution is charged to this account. In this scenario, the customer enters a commercial contract only with the simulation software vendor and it is the vendor's responsibility to provide full customer support.

The selected model (' $a$ ' or ' $b$ ') is a decision made by the simulation software vendor. Please note that the above listed two options may not be the only ones, and further refinement may be required based on the actual use-cases (the options reflect experiences with the project industrial partners). The cloud extended simulation software product can be advertised and offered for download or to be accessed as a web service via the CloudSME AppCenter. However, this association with the CloudSME AppCenter is optional. On the other hand, enabling the simulation through the CSSP is compulsory and an essential requirement. If the simulation does not run through the CSSP, then it is outside the currently described business model. Figure 2(c) illustrates the cloud extension to a desktop or web-based simulation package scenario.

\subsection{Direct Application Execution from the CloudSME Platform}

A simulation software provider (vendor or consultant) may decide not to develop a specialized one-stopshop solution but rather use the CloudSME AppCenter to offer its software as a service offering directly. This scenario is similar to the first business model "specialized one-stop-shop for a specific industry". However, instead of setting up a specific new website, the offered capabilities of the CloudSME AppCenter are utilized. The SaaS solution will be directly executed from the CloudSME AppCenter. The AppCenter provider charges the end-users and takes a certain percentage before paying the application owner. The advantage of this solution is that setting up the offering is quicker and requires much fewer resources. Also, platform related support in this case is provided directly to the simulation end-user by the platform provider (the AppCenter operator). The disadvantage is that the result is far less customized than in the case of the "specialized one-stop-shop for a specific industry" scenario. Also, the level of customer support may be lower as only generic, platform specific support can be expected from the platform provider. In case of large customer numbers, the solution may become unfeasible and the development of a specialized one-stop-shop may be required. This option can be a good entry-point for new potential MSaaS developers in order to test or start marketing their solution while minimizing cost/risk. This business model is shown in Figure 2(d).

\subsection{CloudSME Business Model for Platform Providers}

The final business model reflects the platform provider, in this case the stakeholders supporting the CSSP. The aim is to facilitate a wide range of business models for MSaaS providers and their end-users - the customers of platform. Given the number of stakeholders involved, the simplest business model is based around creating a dedicated company that acts as an entity in its own right. This means that the company 
a)

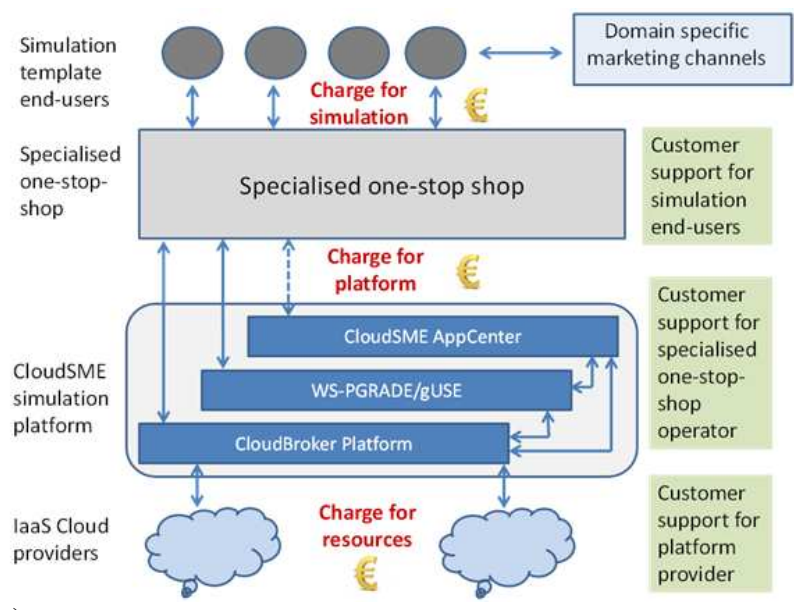

c)

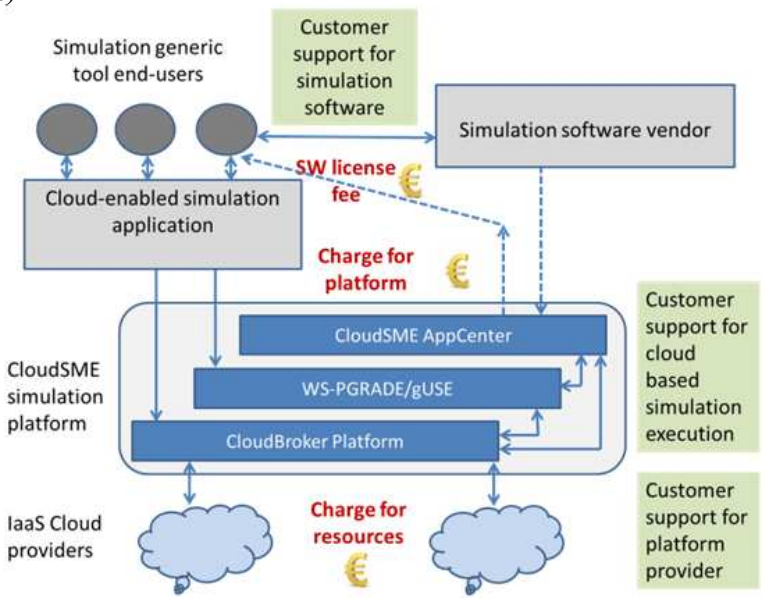

b)

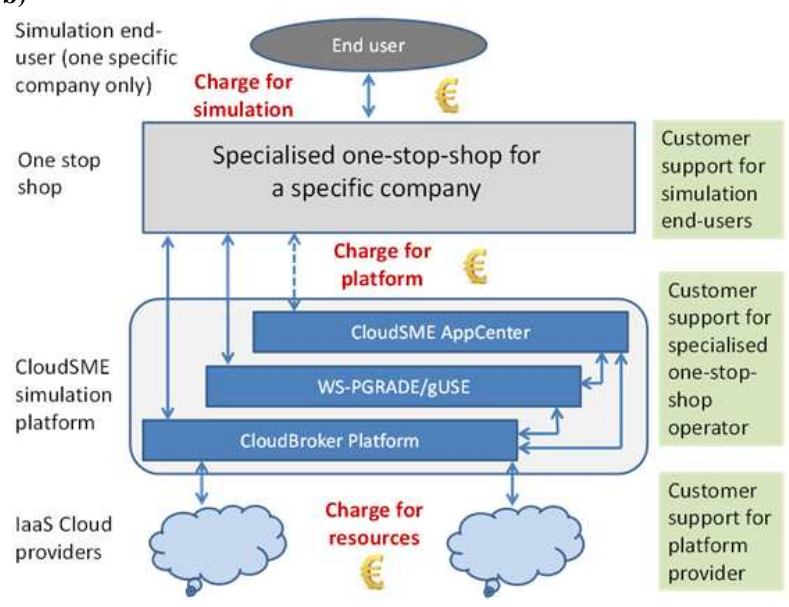

d)

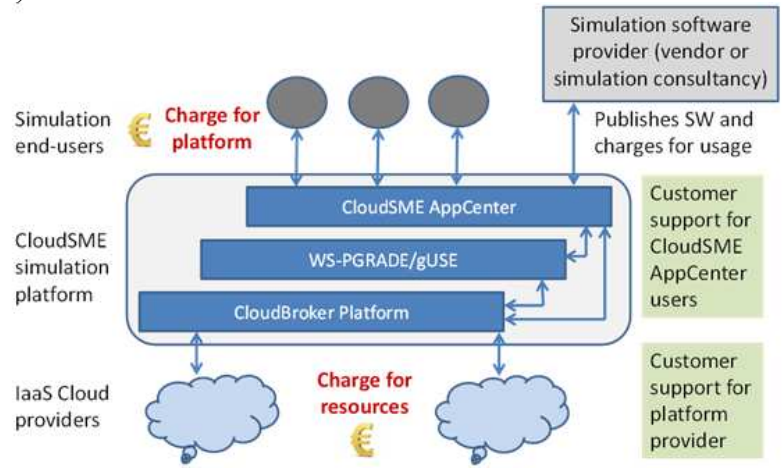

Figure 2: MSaaS Business Models (a) Specialized one-stop-shop for a specific industry, (b) Specialized one-stop-shop for a specific company, (c) Cloud extension to a desktop simulation or a web-based package, and (d) Direct application execution from the CSSP.

charges for platform use, pays for infrastructure resources and then distributes proceeds within the company.

\section{DISCUSSION}

As discussed in this paper, Modeling \& Simulation as Service (MSaaS) enables simulation software vendors and providers to offer simulation applications through the cloud to their end-users. Solutions range from direct use of cloud resources by an application to using more sophisticated chains of technological services involving PaaS and IaaS solutions. The benefit to the end-user is potentially cheaper, more accessible simulation technologies. The benefit to the simulation developer is new business offerings that could give access to new markets, new products and a major competitive edge. The CloudSME Simulation Platform is an example of how PaaS and IaaS can be provided to support the creation of new products based on MSaaS offerings.

The definition adopted in this paper of MSaaS identifies the need to deliver value to customers and to enable or support M\&S applications and capabilities. "Value" is associated with what a service enables a customer to do or as a service that is part of a chain of technologies that deliver that customer service. The 


\section{Kiss, Dagdeviren, Taylor, Anagnostou, and Fantini}

industrial MSaaS development that is part of the CloudSME project has enabled us to understand the variety of business needs related to service delivery and the roles of the many stakeholders that are part of a complex cloud-based business offering. These include end-users, simulation software providers, cloud platform providers and cloud resource providers. Discussions with the stakeholders involved in CloudSME initially generated a general business model that largely reflected the service grouping of the cloud-based solutions, i.e., end-users using MSaaS hosted on a PaaS using IaaS. The business models helped to consolidate the need for the use of a customer interface - the one-stop-shops and AppCenter implemented in the Simulation Application Layer of the CSSP and described above. Five business models were identified that reflected MSaaS implementation and the product offering: a specialized "one-stopshop" for a specific industry; a specialized "one-stop-shop" for a specific company, a cloud extension to a desktop simulation package or a web-based simulation solution, direct execution of the simulation software from the CloudSME AppCenter, and a business model reflecting the platform service provision (PaaS).

The business models help to identify how revenue can be collected and distributed across stakeholders. Essentially, end-users are charged at the point of use by the simulation software providers (or via license, for example). However, after this point the revenue stream becomes less clear. Many different models were considered in the development of the business models presented in the previous section. For example, end-users could pay for cloud infrastructure resources directly. However, it was felt that this would place an unreasonable expectation on the customer to decide whether or not s/he wanted to use Amazon, CloudSigma, etc. and then understand the different charging mechanisms that different cloud infrastructure providers use. Many of the MSaaS solutions that have been developed place the decision at the software vendor level and hide these details from the end-user (to simplify cloud use and maximize benefit). Another business model that was considered was one that reflected the individual stakeholders involved in the provision of the CSSP. This was rejected on grounds of complexity. A simulation software vendor would have to establish commercial relationships between all stakeholders that reflected their contribution to the specific MSaaS product offering. In some cases, this would have involved at least five different contracts with one software vendor. Instead, a simpler model was adopted that brought the stakeholders that provided and supported the platform together into a single legal entity. A single contract could then be issued between the platform company and the software vendor. This company would collect payment for using the platform and the infrastructure, pay the cloud infrastructure provider and then distribute revenue across the company partners on an internally agreed basis.

What is common to all the business models discussed in this paper is the need to collect revenue based on both platform and infrastructure use. In some ways this reinforced the need for an identified entry point to the MSaaS application to enable end-users to be identified and then to be charged on an appropriate basis (pay-per-use, annual license, etc.) This partially motivated the development of the onestop-shop application specific "portals" and the general AppCenter. Users register at a one-stop-shop and use the software. Depending on the company, users either need to pay up front or are invoiced later for use. An identified user then uses the software. This in turn uses the CSSP which has internal charging mechanisms that are used to track a user's use of a given cloud infrastructure. Direct users of the platform are charged in a similar manner. Arguably, this both simplifies charging mechanisms and enables endusers to be presented with a single point of charging rather than point-to-point relationships with the many stakeholders involved in supporting cloud-based simulation services.

\section{CONCLUSIONS}

This paper has presented business models that have been created based on experiences in the CloudSME project. The project focuses on SMEs in manufacturing and engineering who both use and provide simulation services. These business models are not an exhaustive list and new business models are being considered. Further business models and the evolution of those presented in this paper will be reported in 
Kiss, Dagdeviren, Taylor, Anagnostou, and Fantini

future work. Overall, we hope that these reflections will prove useful in identifying and simplifying potentially complex business relationships in the MSaaS business offerings.

\section{ACKNOWLEDGEMENTS}

This work was partially supported by the EU FP7 CloudSME project (Contract no. 608886). The authors thank the CloudSME beneficiaries for their input in developing the business models described in this paper. A full list of companies involved in the project can be found at www.cloudsme.eu.

\section{REFERENCES}

Cayirci, E. 2013. "Modeling and Simulation as a Cloud Service: A Survey." In Proceedings of the 2013 Winter Simulation Conference, edited by R. Pasupathy, S.-H. Kim, A. Tolk, R. Hill, and M. E. Kuhl, 389-400. Piscataway, New Jersey: Institute of Electrical and Electronics Engineers, Inc.

Johnson, H. E., and A. Tolk. 2013. "Evaluating the Applicability of Cloud Computing Enterprises in Support of the Next Generation of Modeling and Simulation Architectures." In Proceedings of the Military Modeling \& Simulation Symposium (MMS '13). Society for Computer Simulation International, San Diego, CA, USA, Article 4, 8 pages.

Kacsuk, P. 2014. Science Gateways for Distributed Computing Infrastructures: Development Framework and Exploitation by Scientific User Communities. Springer International Publishing Switzerland.

Kacsuk, P., Z. Farkas, M. Kozlovszky, G. Hermann, A. Balasko, K. Karoczkai, and I. Marton. 2012. "WS-PGRADE/gUSE Generic DCI Gateway Framework for a Large Variety of User Communities." Journal of Grid Computing 10(4): 601-630.

Kacsuk, P. 2011. "P-GRADE Portal Family for Grid Infrastructures." Concurrency and Computation: Practice and Experience 23(3): 235-245.

Liu, X., X. Qiu, B. Chen, and K. Huang. 2012. "Cloud-Based Simulation: The State-of-the-Art Computer Simulation Paradigm." In Proceedings of the 2012 ACM/IEEE/SCS Workshop on Principles of Advanced and Distributed Simulation (PADS 2012), 71-74. ACM Press, NY.

Luo, J., and L. J. Hong. 2011. "Large-Scale Ranking and Selection Using Cloud Computing." In Proceedings of the 2011 Winter Simulation Conference, edited by S. Jain, R. R. Creasey, J. Himmelspach, K. P. White, and M. Fu, 4046-4056. Piscataway, New Jersey: Institute of Electrical and Electronics Engineers, Inc.

Mell, P. and T. Grance. 2011. The NIST Definition of Cloud Computing. Accessed May 06, 2015. http://csrc.nist.gov/publications/nistpubs/800-145/SP800-145.pdf.

NIST. 2013. NIST Cloud Computing Standards Roadmap. Accessed May 06, 2015. http://www.nist.gov/itl/cloud/upload/NIST_SP-500-291_Version-2_2013_June18_FINAL.pdf.

Rossetti, M. D., and Y. Chen. 2012. "A Cloud Computing Architecture for Supply Chain Network Simulation." In Proceedings of the 2012 Winter Simulation Conference, edited by C. Laroque, J. Himmelspach, R. Pasupathy, O. Rose, and A.M. Uhrmacher, 3225-3236. Piscataway, New Jersey: Institute of Electrical and Electronics Engineers, Inc.

Siegfried, R., T. van den Berg, A. Cramp, and W. Huiskamp. 2014. "M\&S as a Service: Expectations and Challenges." In Proceedings of the 2014 Fall Simulation Interoperability Workshop (14F-SIW-040), Orlando, Florida: Simulation Interoperability Standards Organization.

Taylor, S. J. E., A. Anagnostou, T. Kiss, G. Terstyanszky, P. Kacsuk, and N. Fantini, N. 2014a. "A Tutorial on Cloud Computing for Agent-Based Modeling \& Simulation with REPAST." In Proceedings of the 2014 Winter Simulation Conference, edited by A. Tolk, S. Y. Diallo, I. O. Ryzhov, L. Yilmaz, S. Buckley, and J. A. Miller, 192-206. Piscataway, New Jersey: Institute of Electrical and Electronics Engineers, Inc. 
Taylor, S. J. E., T. Kiss, G. Terstyanszky, P. Kacsuk, and N. Fantini. 2014b. "Cloud Computing for Simulation in Manufacturing and Engineering: Introducing the CloudSME Simulation Platform." In Proceedings of ANSS'14, Tampa, FL, Apr. 13 - 16, Article 12.

Taylor, S. J. E., A. Khan, K. L. Morse, A. Tolk, L. Yilmaz, and J. Zander. 2013a. "Grand Challenges on The Theory of Modeling and Simulation." In Proceedings of the Symposium on Theory of Modeling \& Simulation - DEVS Integrative M\&S Symposium (DEVS'13), Society for Computer Simulation International, San Diego, CA, USA, Article 34,8 pages.

Taylor, S. J. E., S. E. Chick, C. M. Macal, S. Brailsford, P. L'Ecuyer, and B. L. Nelson. 2013b. "Modeling and simulation grand challenges: An OR/MS perspective." In Proceedings of the 2013 Winter Simulation Conference, edited by R. Pasupathy, S.-H. Kim, A. Tolk, R. Hill, and M. E. Kuhl, 12691282. Piscataway, New Jersey: Institute of Electrical and Electronics Engineers, Inc.

Taylor, S. J. E., R. Fujimoto, E. H. Page, P. A. Fishwick, A. M. Uhrmacher, and G. Wainer. 2012. "Panel on Grand Challenges for Modeling and Simulation." In Proceedings of the 2012 Winter Simulation Conference, edited by C. Laroque, J. Himmelspach, R. Pasupathy, O. Rose, and A.M. Uhrmacher, 2614-2628. Piscataway, New Jersey: Institute of Electrical and Electronics Engineers, Inc.

Tolk, A., and Mittal, S. 2014. "A Necessary Paradigm Change to Enable Composable Cloud-Based M\&S Services." In Proceedings of the 2014 Winter Simulation Conference, edited by A. Tolk, S. Y. Diallo, I. O. Ryzhov, L. Yilmaz, S. Buckley, and J. A. Miller, 356-366. Piscataway, New Jersey: Institute of Electrical and Electronics Engineers, Inc.

Wu, X., Qiao, F. and Poon, K. 2014. "Cloud Manufacturing Application in Semiconductor Industry." In Proceedings of the 2014 Winter Simulation Conference, edited by A. Tolk, S. Y. Diallo, I. O. Ryzhov, L. Yilmaz, S. Buckley, and J. A. Miller, 2376-2383. Piscataway, New Jersey: Institute of Electrical and Electronics Engineers, Inc.

\section{AUTHOR BIOGRAPHIES}

TAMAS KISS is a Reader in the Department of Computer Science, Faculty of Science and Technology, University of Westminster. Currently he is Project Director of the FP7 CloudSME project. His email address is T.Kiss@westminster.ac.uk.

HUSEYIN DAGDEVIREN is a Senior Lecturer in the Department of Computer Science, Faculty of Science and Technology, University of Westminster. His email address is H.Dagdeviren@westminster.ac.uk.

SIMON J. E. TAYLOR is a Reader in the Department of Computer Science at Brunel University London and leads the Modelling \& simulation Group as well as leading product development in CloudSME. His email address is simon.taylor@brunel.ac.uk.

ANASTASIA ANAGNOSTOU is a Research Fellow in the Department of Computer Science, Brunel University London. Her email address is anastasia.anagnostou@brunel.ac.uk.

NICOLA FANTINI is the CEO of the CloudBroker $\mathrm{GmbH}, \mathrm{CH}$, and has been software engineer, project manager and entrepreneur in the field of software development for more than 20 years. His email address is nicola.fantini@cloudbroker.com. 